\title{
Benchmark Specification of Advanced Burner Test Reactor
}

Nuclear Science and Engineering Division 
About Argonne National Laboratory

Argonne is a U.S. Department of Energy laboratory managed by UChicago Argonne, LLC under contract DE-AC02-06CH11357. The Laboratory's main facility is outside Chicago, at 9700 South Cass Avenue, Argonne, Illinois 60439. For information about Argonne

and its pioneering science and technology programs, see www.anl.gov.

\section{DOCUMENT AVAILABILITY}

Online Access: U.S. Department of Energy (DOE) reports produced after 1991 and a growing number of pre-1991 documents are available free at OSTI.GOV

(http://www.osti.gov/), a service of the US Dept. of Energy's Office of Scientific and Technical Information.

Reports not in digital format may be purchased by the public from the National Technical Information Service (NTIS):

U.S. Department of Commerce

National Technical Information Service

5301 Shawnee Rd

Alexandria, VA 22312

www.ntis.gov

Phone: (800) 553-NTIS (6847) or (703) 605-6000

Fax: (703) 605-6900

Email: orders@ntis.gov

Reports not in digital format are available to DOE and DOE contractors from the Office of Scientific and Technical Information (OSTI):

U.S. Department of Energy

Office of Scientific and Technical Information

P.O. Box 62

Oak Ridge, TN 37831-0062

www.osti.gov

Phone: (865) 576-8401

Fax: (865) 576-5728

Email: reports@osti.gov

Disclaimer

This report was prepared as an account of work sponsored by an agency of the United States Government. Neither the United States Government nor any agency thereof, nor UChicago Argonne, LLC, nor any of their employees or officers, makes any warranty, express or implied, or assumes any legal liability or responsibility for the accuracy, completeness, or usefulness of any information, apparatus, product, or process disclosed, or represents that its use would not infringe privately owned rights. Reference herein to any specific commercial product, process, or service by trade name, trademark, manufacturer, or otherwise, does not necessarily constitute or imply its endorsement, recommendation, or favoring by the United States Government or any agency thereof. The views and opinions of document authors expressed herein do not necessarily state or reflect those of the United States Government or any agency thereof, Argonne National Laboratory, or UChicago Argonne, LLC 


\section{Benchmark Specification of Advanced Burner Test Reactor}

prepared by

Taek K. Kim

Nuclear Science and Engineering Division, Argonne National Laboratory

December 16, 2020 


\section{Introduction}

As an effort to assess the Argonne Reactor Computation (ARC) suite of fast reactor analysis codes, a numerical benchmark problem was developed using the reference $250 \mathrm{MWt}$ Advanced Burner Test Reactor (ABTR) metallic core fueled with beginning of equilibrium cycle compositions (Chang et al., 2006). Material thermal expansion at operating condition was modeled by adjusting the hexagonal pitch, axial meshes, and the fuel and structure material densities appropriately. Irradiation swelling of metal fuel was considered, and the bond sodium was displaced into the lower part of fission gas plenum.

\section{ABTR Benchmark Core}

Figure 1 shows the core configuration of the $250 \mathrm{MWt}$ ABTR benchmark problems. The core configuration was obtained from the reference ABTR core design (Chang et al., 2006). The inner and outer cores are composed of 24 and 30 driver assemblies, respectively. Nine test assembly locations were introduced in the reference ABTR core design. Among these test locations, three assemblies located in the fourth row were designated for material test assemblies, and six test assemblies located in the third and fourth rows were designated for fuel test assemblies. Since the compositions of the test assemblies are not determined at this point, material test assembly locations were loaded by reflector assemblies, while the fuel test assembly locations were loaded by the driver fuel assemblies.

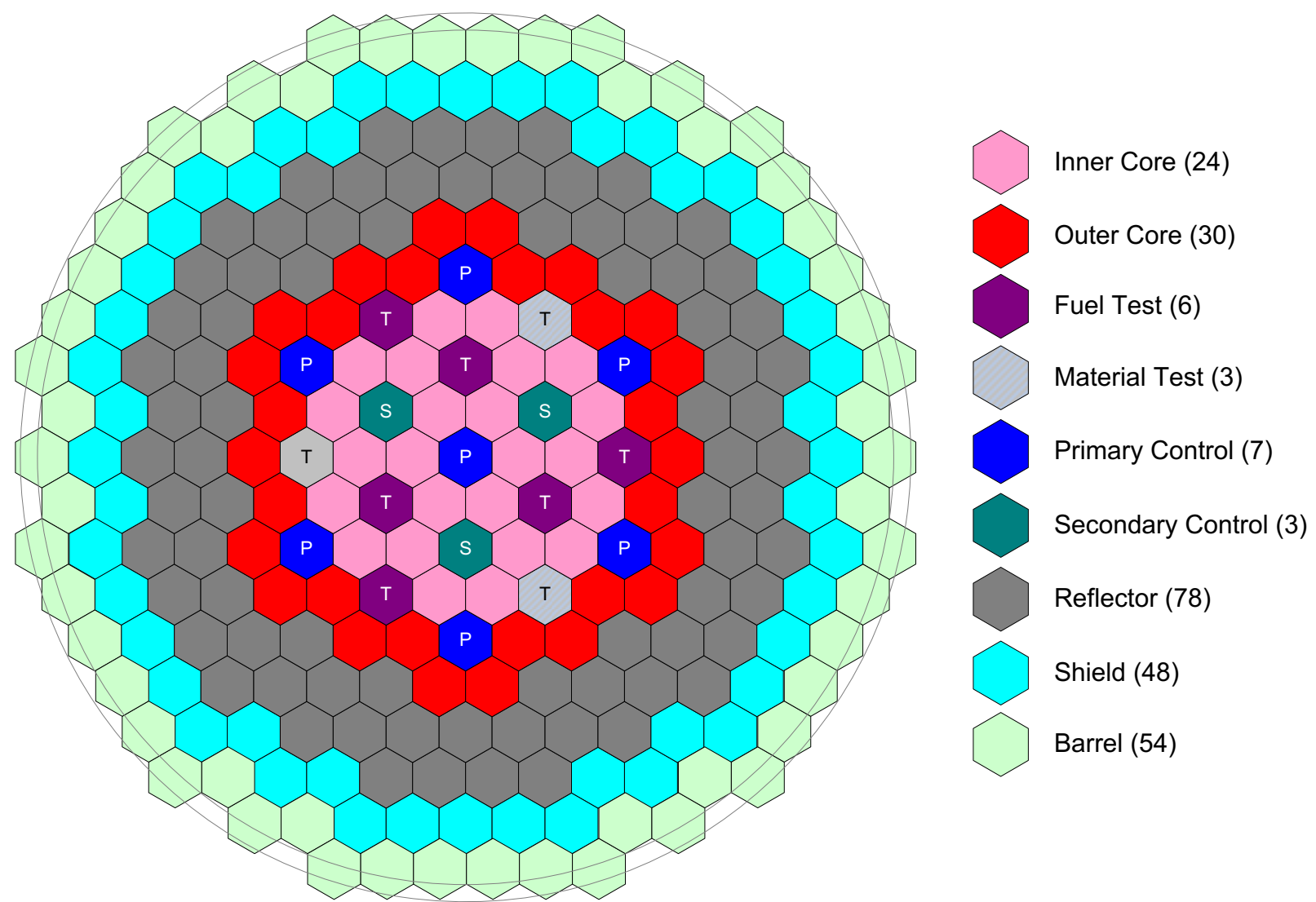

Figure 1 Radial core layout of ABTR benchmark problem 
Two independent safety-grade reactivity control systems are employed in ABTR reactor: primary and secondary control rod systems. The primary control system consists of one central assembly and six assemblies in fifth row, and the secondary control system consists of three assemblies in the third row. In the benchmark problem, all control assemblies are positioned above the active core.

The fuel composition at the equilibrium cycle was obtained from the REBUS-3 calculations using a lumped fission product (LFP) model. It should be emphasized here that the variation fuel compositions were extremely simplified in this benchmark and the lumped fission product was replaced by Molybdenum (Mo) because most code systems do not have the cross sections for the lumped fission product. The material information is provided in Appendix.

The assembly design data of fuel, reflector, shield and control assemblies are presented in Table 1, Table 2, Table 3, and Table 4, respectively. Since the thermal expansions of fuel and supporting material are important in ABTR core analysis, the benchmark problem was developed at the operating condition, including irradiation swelling of metal fuel. For this reason, the sodium bond (between fuel slug and cladding) is replaced to the upper gas plenum. However, the data at the room temperature are also provided for the comparison purpose.

The fuel pin and control rod are helically wrapped with wire to maintain the pin spacing such that the coolant can flow freely through the pin bundle. The wire-wrap was assumed be smeared in clad for the simplicity of the benchmark problem. Due to this reason, the outer radii of the fuel and control rod claddings are slightly increased in the benchmark problem.

At the operating condition, the metal fuel alloy is expanded radially and axially due to the thermal expansion and burnup swelling effect. The metal fuel alloy expands till contacting the inner surface of the fuel clad and glows axially along to the gas plenum, and the sodium bond is displaced to the gas plenum above the fuel. The smear density and axial swelling were assumed to be $75 \%$ and $5 \%$, respectively.

Figure 2, Figure 3, and Figure 4 show the schematics of fuel, control and reflector/shield assemblies, respectively. Dimensions are obtained at the operating condition. All assemblies have the same lower and upper structures. A homogeneous mixture of sodium (70\%) and SS$316(30 \%)$ was assumed as the lower structure. Since the upper structure is so complicate, the upper structure is assumed to be a homogeneous mixture of the lower reflector of the fuel assembly. Table 5 provides the average operating temperatures.

The fuel assembly contains 217 fuel pins arranged in a triangular pitch array. The fuel volume fraction at the operating condition is $31.42 \%$. The reflector assembly contains 91 solid HT-9 pins arranged in a triangular pitch array. The HT-9 pin volume fraction is $75.2 \%$ and the duct volume fraction is $7.7 \%$, yielding the total HT-9 volume fraction of $83.9 \%$ at the operating condition. The shield assembly is mainly composed of HT-9 clad and $\mathrm{B}_{4} \mathrm{C}$ absorber. It consists of 19 thick HT-9 tubes containing boron carbide pellets. The natural boron is used with $81 \%$ of a smeared $\mathrm{B}_{4} \mathrm{C}$ pellet density.

The control assemblies consist of an absorber bundle contained within a duct. The absorber bundle is a closely packed array of tubes containing compacted boron carbide pellets. The bundle of pins is contained in an interior duct that channels flow through the bundle and protects the pins from damage as they slide within the outer fixed duct. The outer duct of the control rod assembly has the same external dimensions as the fuel assembly duct. 
Table 1 Parameters of Fuel Assembly

\begin{tabular}{|l|r|r|}
\hline & Cold & Hot \\
\hline Overall length of duct, cm & 340.000 & 341.663 \\
\hline Number of pins & \multicolumn{2}{|c|}{217} \\
\hline Fuel pin smear density, \% & 75 & \\
\hline Fuel pin pitch, cm & 0.9080 & 0.9134 \\
\hline Fuel pin diameter, cm & 0.8000 & 0.8048 \\
\hline Thickness of clad, cm & 0.0520 & 0.0523 \\
\hline Outer radius of clad, cm & 0.4000 & 0.4024 \\
\hline Inner radius of clad, cm & 0.3480 & 0.3501 \\
\hline Fuel slug radius, cm & 0.3014 & 0.3501 \\
\hline Active core height, cm & 80.0000 & 84.4108 \\
\hline Gas plenum height, cm & 120.0000 & 120.5868 \\
\hline Assembly pitch, cm & 14.5980 & 14.6850 \\
\hline Duct outer Flat-to-Flat distance, cm & 14.1980 & 14.2826 \\
\hline Duct wall thickness, cm & 0.3000 & 0.3018 \\
\hline Duct inside flat-to-flat distance, $\mathrm{cm}$ & 13.5980 & 13.6790 \\
\hline Wire wrap radius, cm & 0.0515 & \\
\hline Wire wrap axial pitch, cm & 20.3200 & \\
\hline Clad outer radius with wire-wrap, cm & & 0.4057 \\
\hline Lower reflector height, cm & 60.000 & 60.293 \\
\hline length of displaced sodium bond, cm & 0.0000 & 19.7637 \\
\hline Volume fraction & & \\
- Fuel & 0.3355 & 0.3142 \\
- Clad & 0.1536 & 0.1510 \\
- Duct & 0.0783 & 0.0770 \\
- Sodium bond & 0.1118 & 0.0000 \\
- Coolant & 0.3208 & 0.3208 \\
\hline
\end{tabular}


Table 2 Parameters of Reflector Assembly

\begin{tabular}{|l|l|l|}
\hline & Cold & Hot \\
\hline Overall length of duct, cm & 340.000 & 341.663 \\
\hline Number of pins & 91 & \multicolumn{2}{|l|}{} \\
\hline Pin pitch, cm & 1.4067 & 1.4151 \\
\hline Reflector rod diameter, cm & 1.4052 & 1.4136 \\
\hline Reflector rod radius, cm & 0.7026 & 0.7068 \\
\hline Reflector rod height, cm & 260.0000 & 261.2714 \\
\hline Assembly pitch, cm & 14.5980 & 14.6850 \\
\hline Duct outer flat-to-flat distance, cm & 14.1980 & 14.2826 \\
\hline Duct wall thickness, cm & 0.3000 & 0.3018 \\
\hline Duct inside flat-to-flat distance, cm & 13.5980 & 13.6790 \\
\hline Volume fraction & & \\
- Fuel & 0.76470 & 0.75199 \\
- Duct & 0.07826 & 0.07696 \\
- - Coolant & 0.15704 & 0.15704 \\
\hline
\end{tabular}

Table 3 Parameters of Shield Assembly

\begin{tabular}{|l|l|l|}
\hline & Cold & Hot \\
\hline Overall length of duct, cm & 340.000 & 341.663 \\
\hline Number of pins & 19 & \\
\hline Shield pin pitch, cm & 3.0441 & 3.0622 \\
\hline Absorber smear density, \% & 81 & \\
\hline Shield pin diameter, cm & 3.0426 & 3.0607 \\
\hline Thickness of clad, cm & 0.2500 & 0.2515 \\
\hline Outer radius of clad, cm & 1.5213 & 1.5304 \\
\hline Inner radius of clad, cm & 1.2713 & 1.2789 \\
\hline Absorber radius, cm & 1.1442 & 1.2789 \\
\hline Absorber height, cm & 260.0000 & 261.2714 \\
\hline Assembly pitch, cm & 14.5980 & 14.6850 \\
\hline Duct outer flat-to-flat distance, cm & 14.1980 & 14.2826 \\
\hline Duct wall thickness, cm & 0.3000 & 0.3018 \\
\hline Duct inside flat-to-flat distance, cm & 13.5980 & 13.6790 \\
\hline Volume fraction & & \\
- Fuel & 0.42341 & 0.41637 \\
- Clad & 0.22580 & 0.22050 \\
- Duct & 0.07826 & 0.07696 \\
- He bond & 0.09932 & 0.00000 \\
- Coolant & 0.17320 & 0.17320 \\
\hline
\end{tabular}


Table 4 Parameters of Control Assembly

\begin{tabular}{|l|l|l|}
\hline & Cold & Hot \\
\hline Overall length of duct, cm & 340.000 & 341.663 \\
\hline Number of pins & 91 & \\
\hline Rod pitch & 1.2484 & 1.2558 \\
\hline Absorber smear density, \% & 85 & \\
\hline pin diameter, cm & 1.1104 & 1.1170 \\
\hline Thickness of clad, cm & 0.0700 & 0.0704 \\
\hline Outer radius of clad, cm & 0.5552 & 0.5585 \\
\hline Inner radius of clad, cm & 0.4852 & 0.4881 \\
\hline Absorber radius, cm & 0.4473 & 0.4881 \\
\hline Absorber height, cm & 85.0000 & 85.4157 \\
\hline Assembly pitch, cm & 14.5980 & 14.6850 \\
\hline Duct outer flat-to-flat distance, cm & 14.1980 & 14.2826 \\
\hline Duct wall thickness, cm & 0.3000 & 0.3018 \\
\hline Duct inside flat-to-flat distance, cm & 13.5980 & 13.6790 \\
\hline Inner duct outer flat-to-flat distance, cm & 12.7980 & 12.8743 \\
\hline Inner duct inner flat-to-flat distance, cm & 12.1980 & 12.2707 \\
\hline Wire wrap radius, cm & 0.0665 & \\
\hline Wire wrap axial pitch, cm & 20.3200 & \\
\hline Clad outer radius with wire-wrap & & 0.5626 \\
\hline Radius of follower & 5.0000 & 5.0298 \\
\hline Length of follower & 16.8000 & 16.8822 \\
\hline Volume fraction & & \\
- Fuel & 0.30996 & 0.30481 \\
- Clad & 0.11979 & 0.11780 \\
- Duct & 0.14864 & 0.14617 \\
- He bond & 0.05470 & 0.00000 \\
- Coolant & 0.36691 & 0.36692 \\
\hline
\end{tabular}

Table 5 Temperature at Operating Condition

\begin{tabular}{|l|l|}
\hline & Temperature $\left({ }^{\circ} \mathrm{C}\right)$ \\
\hline Active core & \\
Coolant & 432.5 \\
Structure & 462.5 \\
Fuel & 582.5 \\
\hline Reflector & 432.5 \\
\hline Shield & 355.0 \\
\hline Lower structure and reflector & 355.0 \\
\hline Upper plenum and structure & 510.0 \\
\hline
\end{tabular}



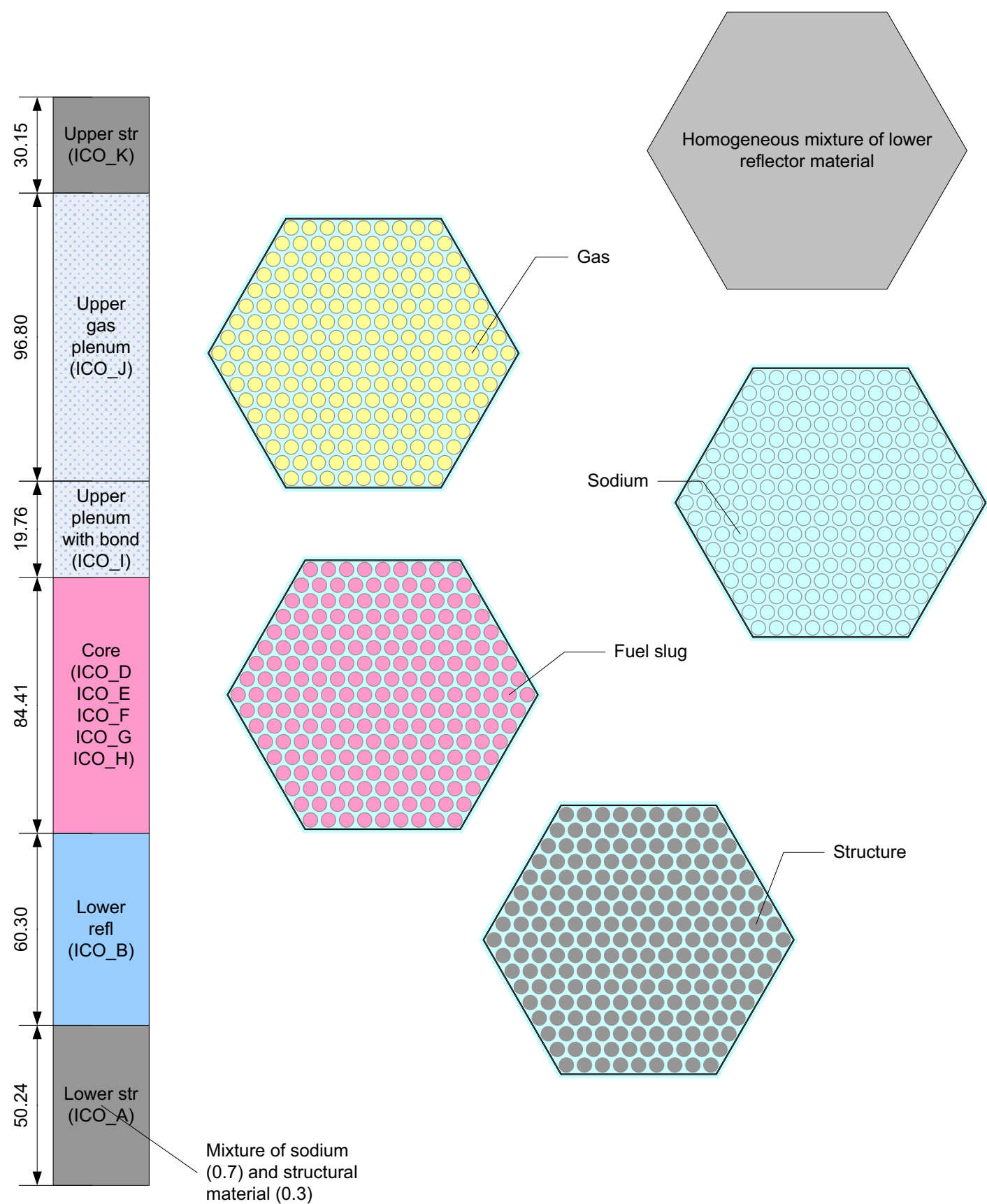

Figure 2 Fuel Assembly Schematics 

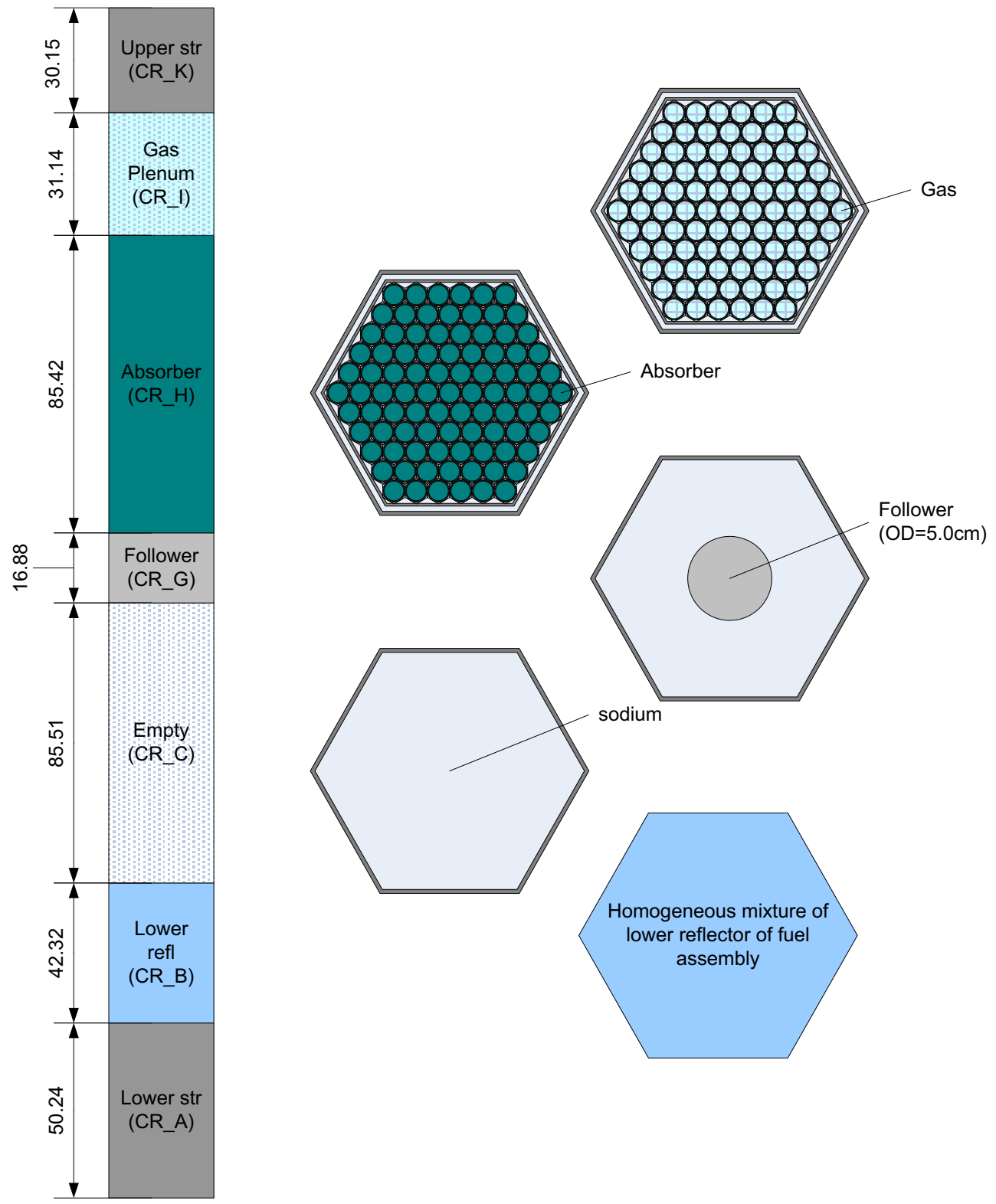

Figure 3 Control Assembly Schematics 

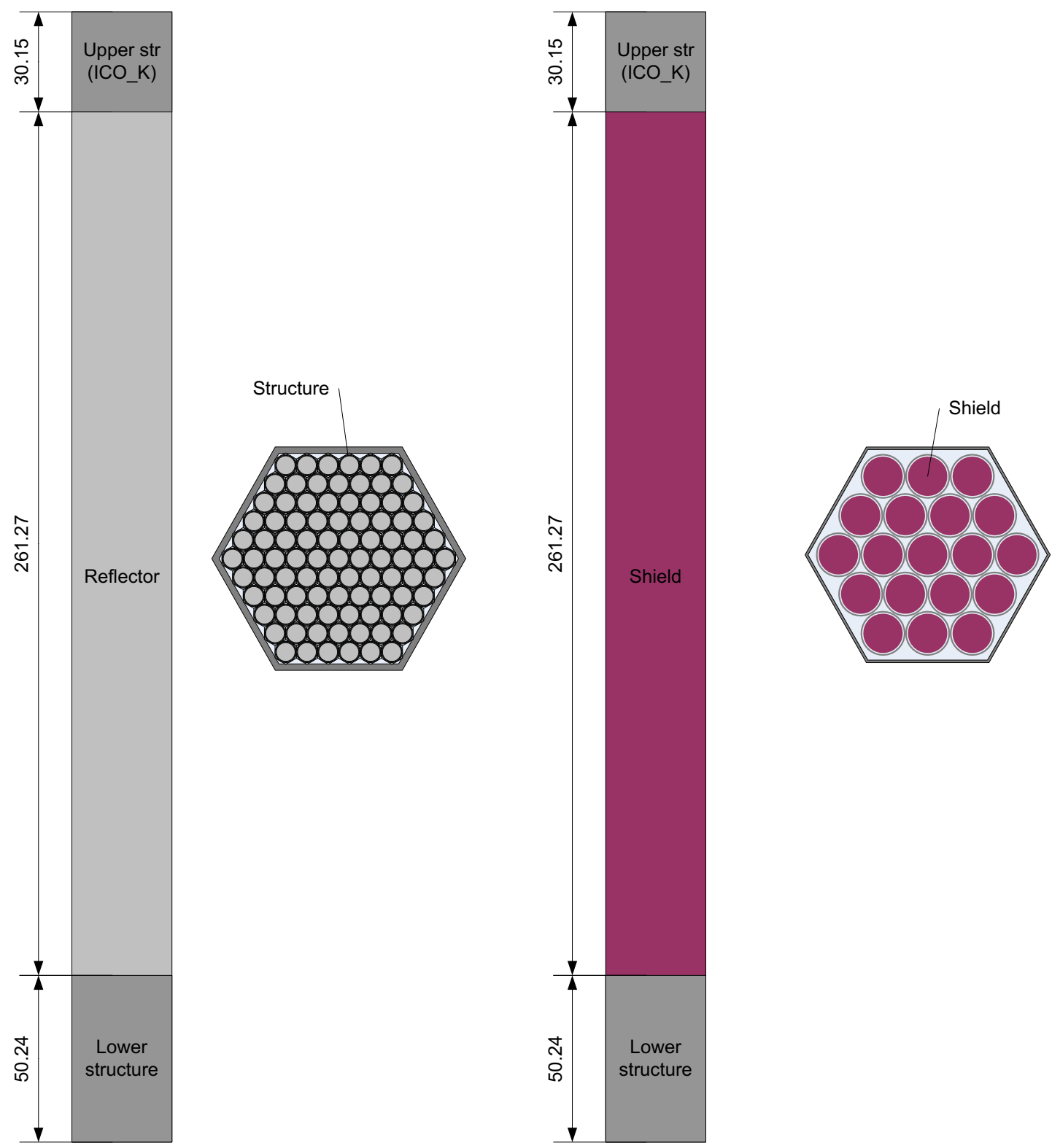

Figure 4 Radial Reflector/Shield Assembly Schematics 


\section{Reference}

Chang, Y. I., et al. (2006), "Advanced Burner Test Reactor Preconceptual Design Report," ANLABR-1, ANL-AFCl-173, Argonne National Laboratory, September 5, 2006. 


\section{Appendix Composition Number Densities (/barn-cm)}

1. Lower Structure

\begin{tabular}{|l|l|}
\hline $\mathrm{Na}$ & $1.5591 \mathrm{E}-02$ \\
\hline $\mathrm{Fe}$ & $1.5878 \mathrm{E}-02$ \\
\hline $\mathrm{Ni}$ & $3.2604 \mathrm{E}-03$ \\
\hline $\mathrm{Cr}$ & $3.2355 \mathrm{E}-03$ \\
\hline $\mathrm{Mn} 55$ & $5.0846 \mathrm{E}-04$ \\
\hline $\mathrm{Mo}$ & $4.3524 \mathrm{E}-04$ \\
\hline
\end{tabular}

2. Lower Reflector of fuel assembly

\begin{tabular}{|l|l|l|l|l|}
\hline & \multicolumn{3}{|l|}{ Heterogeneous } & Homogeneou \\
& Clad \& str. & Coolant & Duct & S \\
\hline $\mathrm{Na}$ & & $2.2272 \mathrm{E}-02$ & & $7.1455 \mathrm{E}-03$ \\
\hline $\mathrm{Fe}$ & $6.9844 \mathrm{E}-02$ & & $6.9844 \mathrm{E}-02$ & $4.7436 \mathrm{E}-02$ \\
\hline $\mathrm{Ni}$ & $4.3063 \mathrm{E}-04$ & & $4.3063 \mathrm{E}-04$ & $2.9248 \mathrm{E}-04$ \\
\hline $\mathrm{Cr}$ & $1.0385 \mathrm{E}-02$ & & $1.0385 \mathrm{E}-02$ & $7.0532 \mathrm{E}-03$ \\
\hline $\mathrm{Mn} 55$ & $4.6007 \mathrm{E}-04$ & & $4.6007 \mathrm{E}-04$ & $3.1247 \mathrm{E}-04$ \\
\hline $\mathrm{Mo}$ & $4.9097 \mathrm{E}-04$ & & $4.9097 \mathrm{E}-04$ & $3.3346 \mathrm{E}-04$ \\
\hline
\end{tabular}

3. Active Fuel

\begin{tabular}{|l|l|l|l|l|l|l|}
\hline & \multicolumn{2}{l}{ Heterogeneous } & Clad & Coolant \\
Nuclide & inner core & $\begin{array}{l}\text { middle } \\
\text { core }\end{array}$ & outer core & HT9 & $\begin{array}{l}\text { Duct } \\
\text { Sodium }\end{array}$ & HT9 \\
\hline U-234 & $1.2225 \mathrm{E}-08$ & $1.0027 \mathrm{E}-06$ & $1.4997 \mathrm{E}-08$ & & & \\
U-235 & $3.2248 \mathrm{E}-05$ & $3.2038 \mathrm{E}-05$ & $3.1767 \mathrm{E}-05$ & & \\
U-236 & $2.0561 \mathrm{E}-06$ & $1.9484 \mathrm{E}-06$ & $1.8593 \mathrm{E}-06$ & & \\
U-238 & $2.0222 \mathrm{E}-02$ & $1.9745 \mathrm{E}-02$ & $1.9317 \mathrm{E}-02$ & & \\
Np237 & $3.8387 \mathrm{E}-06$ & $1.8093 \mathrm{E}-04$ & $3.1274 \mathrm{E}-06$ & & \\
Pu236 & $1.3918 \mathrm{E}-11$ & $9.0759 \mathrm{E}-10$ & $9.1074 \mathrm{E}-12$ & & \\
Pu238 & $9.5854 \mathrm{E}-07$ & $9.0509 \mathrm{E}-05$ & $9.8743 \mathrm{E}-07$ & & \\
Pu239 & $3.4991 \mathrm{E}-03$ & $2.3546 \mathrm{E}-03$ & $4.3068 \mathrm{E}-03$ & & \\
Pu240 & $3.7398 \mathrm{E}-04$ & $1.0881 \mathrm{E}-03$ & $4.5617 \mathrm{E}-04$ & & \\
Pu241 & $2.4535 \mathrm{E}-05$ & $2.9432 \mathrm{E}-04$ & $2.9545 \mathrm{E}-05$ & & \\
Pu242 & $1.7542 \mathrm{E}-06$ & $2.1919 \mathrm{E}-04$ & $2.0615 \mathrm{E}-06$ & & \\
Am241 & $1.4209 \mathrm{E}-06$ & $2.1306 \mathrm{E}-04$ & $2.2274 \mathrm{E}-06$ & & \\
Am242 & $2.8475 \mathrm{E}-08$ & $5.8336 \mathrm{E}-06$ & $4.0761 \mathrm{E}-08$ & & \\
m & & & & \\
Am243 & $6.1338 \mathrm{E}-08$ & $4.3624 \mathrm{E}-05$ & $7.5588 \mathrm{E}-08$ & & \\
Cm242 & $4.7083 \mathrm{E}-08$ & $7.3545 \mathrm{E}-06$ & $5.6294 \mathrm{E}-08$ & & \\
Cm243 & $7.4138 \mathrm{E}-10$ & $2.4428 \mathrm{E}-07$ & $9.0540 \mathrm{E}-10$ & & \\
Cm244 & $4.8305 \mathrm{E}-09$ & $1.0986 \mathrm{E}-05$ & $5.5302 \mathrm{E}-09$ & & & \\
Cm245 & $1.9064 \mathrm{E}-10$ & $9.7716 \mathrm{E}-07$ & $1.9972 \mathrm{E}-10$ & & & \\
& & & & & \\
& & & & & \\
\end{tabular}




\begin{tabular}{|c|c|c|c|c|c|c|}
\hline $\mathrm{Cm} 246$ & $2.6112 \mathrm{E}-12$ & $6.7680 \mathrm{E}-08$ & $2.4070 \mathrm{E}-12$ & & & \\
\hline $\mathrm{Zr}$ & $7.2938 \mathrm{E}-03$ & $7.2938 \mathrm{E}-03$ & $7.2938 \mathrm{E}-03$ & & & \\
\hline $\mathrm{Na}$ & & & & & $2.2272 \mathrm{E}-02$ & \\
\hline $\mathrm{Fe}$ & & & & $6.9844 \mathrm{E}-02$ & & $6.9844 \mathrm{E}-02$ \\
\hline $\mathrm{Ni}$ & & & & 4.3063E-04 & & $4.3063 \mathrm{E}-04$ \\
\hline $\mathrm{Cr}$ & & & & $1.0385 \mathrm{E}-02$ & & $1.0385 \mathrm{E}-02$ \\
\hline Mn55 & & & & 4.6007E-04 & & $4.6007 \mathrm{E}-04$ \\
\hline Mo & $\begin{array}{l}9.76123 E- \\
04\end{array}$ & $\begin{array}{l}8.36399 E- \\
04\end{array}$ & $\begin{array}{l}9.81845 E- \\
04\end{array}$ & 4.9097E-04 & & 4.9097E-04 \\
\hline
\end{tabular}

\begin{tabular}{|l|l|l|l|}
\hline & \multicolumn{3}{l}{ Homogeneous } \\
Nuclide & Inner core & middle core & outer core \\
\hline U-234 & $5.46898 \mathrm{E}-09$ & $4.48578 \mathrm{E}-07$ & $6.70877 \mathrm{E}-09$ \\
$\mathrm{U}-235$ & $1.44262 \mathrm{E}-05$ & $1.43324 \mathrm{E}-05$ & $1.42111 \mathrm{E}-05$ \\
$\mathrm{U}-236$ & $9.19798 \mathrm{E}-07$ & $8.71638 \mathrm{E}-07$ & $8.31760 \mathrm{E}-07$ \\
$\mathrm{U}-238$ & $9.04638 \mathrm{E}-03$ & $8.83280 \mathrm{E}-03$ & $8.64147 \mathrm{E}-03$ \\
Np237 & $1.71727 \mathrm{E}-06$ & $8.09410 \mathrm{E}-05$ & $1.39907 \mathrm{E}-06$ \\
Pu236 & $6.22636 \mathrm{E}-12$ & $4.06014 \mathrm{E}-10$ & $4.07421 \mathrm{E}-12$ \\
Pu238 & $4.28807 \mathrm{E}-07$ & $4.04893 \mathrm{E}-05$ & $4.41730 \mathrm{E}-07$ \\
Pu239 & $1.56532 \mathrm{E}-03$ & $1.05336 \mathrm{E}-03$ & $1.92666 \mathrm{E}-03$ \\
Pu240 & $1.67301 \mathrm{E}-04$ & $4.86743 \mathrm{E}-04$ & $2.04069 \mathrm{E}-04$ \\
Pu241 & $1.09760 \mathrm{E}-05$ & $1.31667 \mathrm{E}-04$ & $1.32172 \mathrm{E}-05$ \\
Pu242 & $7.84751 \mathrm{E}-07$ & $9.80540 \mathrm{E}-05$ & $9.22204 \mathrm{E}-07$ \\
Am241 & $6.35636 \mathrm{E}-07$ & $9.53142 \mathrm{E}-05$ & $9.96453 \mathrm{E}-07$ \\
Am242m & $1.27384 \mathrm{E}-08$ & $2.60969 \mathrm{E}-06$ & $1.82346 \mathrm{E}-08$ \\
Am243 & $2.74399 \mathrm{E}-08$ & $1.95154 \mathrm{E}-05$ & $3.38145 \mathrm{E}-08$ \\
$\mathrm{Cm} 242$ & $2.10628 \mathrm{E}-08$ & $3.29008 \mathrm{E}-06$ & $2.51831 \mathrm{E}-08$ \\
$\mathrm{Cm} 243$ & $3.31661 \mathrm{E}-10$ & $1.09281 \mathrm{E}-07$ & $4.05034 \mathrm{E}-10$ \\
$\mathrm{Cm} 244$ & $2.16096 \mathrm{E}-09$ & $4.91452 \mathrm{E}-06$ & $2.47394 \mathrm{E}-09$ \\
$\mathrm{Cm} 245$ & $8.52838 \mathrm{E}-11$ & $4.37138 \mathrm{E}-07$ & $8.93442 \mathrm{E}-11$ \\
$\mathrm{Cm} 246$ & $1.16811 \mathrm{E}-12$ & $3.02767 \mathrm{E}-08$ & $1.07680 \mathrm{E}-12$ \\
$\mathrm{Zr}$ & $3.26289 \mathrm{E}-03$ & $3.26289 \mathrm{E}-03$ & $3.26289 \mathrm{E}-03$ \\
$\mathrm{Na}$ & $7.14528 \mathrm{E}-03$ & $7.14528 \mathrm{E}-03$ & $7.14528 \mathrm{E}-03$ \\
$\mathrm{Fe}$ & $1.61921 \mathrm{E}-02$ & $1.61921 \mathrm{E}-02$ & $1.61921 \mathrm{E}-02$ \\
$\mathrm{Ni}$ & $9.98349 \mathrm{E}-05$ & $9.98349 \mathrm{E}-05$ & $9.98349 \mathrm{E}-05$ \\
$\mathrm{Cr}$ & $2.40756 \mathrm{E}-03$ & $2.40756 \mathrm{E}-03$ & $2.40756 \mathrm{E}-03$ \\
$\mathrm{Mn} 55$ & $1.06659 \mathrm{E}-04$ & $1.06659 \mathrm{E}-04$ & $1.06659 \mathrm{E}-04$ \\
$\mathrm{Mo}$ & $5.50492 \mathrm{E}-04$ & $4.87986 \mathrm{E}-04$ & $5.53051 \mathrm{E}-04$ \\
\hline
\end{tabular}

- Na density of homogeneous voided case is $1.2039 \mathrm{E}-3$ 
4. Gas plenum of fuel assembly with displaced sodium bond

\begin{tabular}{|l|l|l|l|l|}
\hline & \multicolumn{3}{|l|}{ Heterogeneous } & \multirow{2}{*}{ Homogeneous } \\
\hline & Clad & Coolant & Duct & \\
\hline $\mathrm{Na}$ & & $2.2272 \mathrm{E}-02$ & & $1.7109 \mathrm{E}-02$ \\
\hline $\mathrm{Fe}$ & $6.9844 \mathrm{E}-02$ & & $6.9844 \mathrm{E}-02$ & $1.6192 \mathrm{E}-02$ \\
\hline $\mathrm{Ni}$ & $4.3063 \mathrm{E}-04$ & & $4.3063 \mathrm{E}-04$ & $9.9831 \mathrm{E}-05$ \\
\hline $\mathrm{Cr}$ & $1.0385 \mathrm{E}-02$ & & $1.0385 \mathrm{E}-02$ & $2.4075 \mathrm{E}-03$ \\
\hline $\mathrm{Mn} 55$ & $4.6007 \mathrm{E}-04$ & & $4.6007 \mathrm{E}-04$ & $1.0666 \mathrm{E}-04$ \\
\hline $\mathrm{Mo}$ & $4.9097 \mathrm{E}-04$ & & $4.9097 \mathrm{E}-04$ & $1.1382 \mathrm{E}-04$ \\
\hline
\end{tabular}

- Na density of homogeneous voided case is 1.1167E-2

5. Gas plenum of fuel assembly without displaced sodium bond

\begin{tabular}{|l|l|l|l|l|}
\hline & \multicolumn{3}{|l|}{ Heterogeneous } & \multirow{2}{*}{ Homogeneous } \\
\hline & Clad & Coolant & Duct & \\
\hline $\mathrm{Na}$ & & $2.2272 \mathrm{E}-02$ & & $7.1455 \mathrm{E}-03$ \\
\hline $\mathrm{Fe}$ & $6.9844 \mathrm{E}-02$ & & $6.9844 \mathrm{E}-02$ & $1.6192 \mathrm{E}-02$ \\
\hline $\mathrm{Ni}$ & $4.3063 \mathrm{E}-04$ & & $4.3063 \mathrm{E}-04$ & $9.9831 \mathrm{E}-05$ \\
\hline $\mathrm{Cr}$ & $1.0385 \mathrm{E}-02$ & & $1.0385 \mathrm{E}-02$ & $2.4075 \mathrm{E}-03$ \\
\hline $\mathrm{Mn} 55$ & $4.6007 \mathrm{E}-04$ & & $4.6007 \mathrm{E}-04$ & $1.0666 \mathrm{E}-04$ \\
\hline $\mathrm{Mo}$ & $4.9097 \mathrm{E}-04$ & & $4.9097 \mathrm{E}-04$ & $1.1382 \mathrm{E}-04$ \\
\hline
\end{tabular}

- Na density of homogeneous voided case is $1.2039 \mathrm{E}-3$

\section{Empty under Control Rod}

\begin{tabular}{|l|l|l|l|}
\hline & \multicolumn{2}{|l|}{ Heterogeneous } & \multirow{2}{*}{ Homogeneous } \\
\cline { 1 - 3 } Nuclide & Coolant & Duct & \\
\hline $\mathrm{Na}$ & $2.2272 \mathrm{E}-02$ & & $2.0529 \mathrm{E}-02$ \\
\hline $\mathrm{Fe}$ & & $6.9844 \mathrm{E}-02$ & $5.4661 \mathrm{E}-03$ \\
\hline $\mathrm{Ni}$ & & $4.3063 \mathrm{E}-04$ & $3.3702 \mathrm{E}-05$ \\
\hline $\mathrm{Cr}$ & & $1.0385 \mathrm{E}-02$ & $8.1273 \mathrm{E}-04$ \\
\hline $\mathrm{Mn} 55$ & & $4.6007 \mathrm{E}-04$ & $3.6006 \mathrm{E}-05$ \\
\hline $\mathrm{Mo}$ & & $4.9097 \mathrm{E}-04$ & $3.8424 \mathrm{E}-05$ \\
\hline
\end{tabular}


7. Control rod follower

\begin{tabular}{|l|l|l|l|l|}
\hline & \multicolumn{3}{l|}{ Heterogeneous } & \multirow{2}{*}{ Homogeneous } \\
\cline { 1 - 4 } Nuclide & Follower & Coolant & Duct & \\
\cline { 1 - 2 } $\mathrm{Na}$ & & $\begin{array}{l}2.22724 \mathrm{E}- \\
02\end{array}$ & & $1.1051 \mathrm{E}-02$ \\
\hline $\mathrm{Fe}$ & $5.2048 \mathrm{E}-02$ & & $6.9844 \mathrm{E}-02$ & $2.7616 \mathrm{E}-02$ \\
\hline $\mathrm{Ni}$ & $1.0687 \mathrm{E}-02$ & & $4.3063 \mathrm{E}-04$ & $4.5819 \mathrm{E}-03$ \\
\hline $\mathrm{Cr}$ & $1.0606 \mathrm{E}-02$ & & $1.0385 \mathrm{E}-02$ & $5.3263 \mathrm{E}-03$ \\
\hline $\mathrm{Mn55}$ & $1.6667 \mathrm{E}-03$ & & $4.6007 \mathrm{E}-04$ & $7.4530 \mathrm{E}-04$ \\
\hline $\mathrm{Mo}$ & $1.4267 \mathrm{E}-03$ & & $4.9097 \mathrm{E}-04$ & $6.4558 \mathrm{E}-04$ \\
\hline
\end{tabular}

8. Control rod

\begin{tabular}{|l|l|l|l|l|l|}
\hline \multicolumn{5}{|l|}{ Heterogeneous } & \multirow{2}{*}{ Homogeneous } \\
\cline { 1 - 5 } Nuclide & Absorber & Clad & Coolant & Duct & \\
\hline $\mathrm{C}$ & $4.0679 \mathrm{E}-03$ & & & & $1.4834 \mathrm{E}-03$ \\
\hline $\mathrm{B} 10$ & $1.6441 \mathrm{E}-02$ & & & & $5.9956 \mathrm{E}-03$ \\
\hline $\mathrm{B} 11$ & $6.6179 \mathrm{E}-02$ & & & & $2.4133 \mathrm{E}-02$ \\
\hline $\mathrm{Na}$ & & & $2.2272 \mathrm{E}-02$ & & $8.1722 \mathrm{E}-03$ \\
\hline $\mathrm{Fe}$ & & $6.9844 \mathrm{E}-02$ & & $6.9844 \mathrm{E}-02$ & $1.8747 \mathrm{E}-02$ \\
\hline $\mathrm{Ni}$ & & $4.3063 \mathrm{E}-04$ & & $4.3063 \mathrm{E}-04$ & $1.1559 \mathrm{E}-04$ \\
\hline $\mathrm{Cr}$ & & $1.0385 \mathrm{E}-02$ & & $1.0385 \mathrm{E}-02$ & $2.7875 \mathrm{E}-03$ \\
\hline $\mathrm{Mn} 55$ & & $4.6007 \mathrm{E}-04$ & & $4.6007 \mathrm{E}-04$ & $1.2349 \mathrm{E}-04$ \\
\hline $\mathrm{Mo}$ & & $4.9097 \mathrm{E}-04$ & & $4.9097 \mathrm{E}-04$ & $1.3179 \mathrm{E}-04$ \\
\hline
\end{tabular}

9. Gas plenum of control rod

\begin{tabular}{|l|l|l|l|l|}
\hline \multicolumn{4}{|l|}{ Heterogeneous } & \multirow{2}{*}{ Homogeneous } \\
\cline { 1 - 4 } Nuclide & Clad & Coolant & Duct & \\
\hline $\mathrm{Na}$ & & $2.2272 \mathrm{E}-02$ & & $8.1722 \mathrm{E}-03$ \\
\hline $\mathrm{Fe}$ & $6.9844 \mathrm{E}-02$ & & $6.9844 \mathrm{E}-02$ & $1.8747 \mathrm{E}-02$ \\
\hline $\mathrm{Ni}$ & $4.3063 \mathrm{E}-04$ & & $4.3063 \mathrm{E}-04$ & $1.1559 \mathrm{E}-04$ \\
\hline $\mathrm{Cr}$ & $1.0385 \mathrm{E}-02$ & & $1.0385 \mathrm{E}-02$ & $2.7875 \mathrm{E}-03$ \\
\hline $\mathrm{Mn} 55$ & $4.6007 \mathrm{E}-04$ & & $4.6007 \mathrm{E}-04$ & $1.2349 \mathrm{E}-04$ \\
\hline $\mathrm{Mo}$ & $4.9097 \mathrm{E}-04$ & & $4.9097 \mathrm{E}-04$ & $1.3179 \mathrm{E}-04$ \\
\hline
\end{tabular}


10. Reflector

\begin{tabular}{|l|l|l|l|l|}
\hline \multicolumn{4}{|l|}{ Heterogeneous } & \multirow{2}{*}{ Homogeneous } \\
\cline { 1 - 4 } Nuclide & solid rod & Coolant & Duct & \\
\hline $\mathrm{Na}$ & & $2.2272 \mathrm{E}-02$ & & $3.4976 \mathrm{E}-03$ \\
\hline $\mathrm{Fe}$ & $6.9844 \mathrm{E}-02$ & & $6.9844 \mathrm{E}-02$ & $5.8876 \mathrm{E}-02$ \\
\hline $\mathrm{Ni}$ & $4.3063 \mathrm{E}-04$ & & $4.3063 \mathrm{E}-04$ & $3.6301 \mathrm{E}-04$ \\
\hline $\mathrm{Cr}$ & $1.0385 \mathrm{E}-02$ & & $1.0385 \mathrm{E}-02$ & $8.7541 \mathrm{E}-03$ \\
\hline $\mathrm{Mn} 55$ & $4.6007 \mathrm{E}-04$ & & $4.6007 \mathrm{E}-04$ & $3.8782 \mathrm{E}-04$ \\
\hline $\mathrm{Mo}$ & $4.9097 \mathrm{E}-04$ & & $4.9097 \mathrm{E}-04$ & $4.1387 \mathrm{E}-04$ \\
\hline
\end{tabular}

11. Shield

\begin{tabular}{|l|l|l|l|l|l|}
\hline \multicolumn{4}{|l|}{ Heterogeneous } & \multirow{2}{*}{ Homogeneous } \\
\hline Nuclide & Absorber & Clad & Coolant & Duct & \\
\hline C & $3.7154 \mathrm{E}-03$ & & & & $1.9422 \mathrm{E}-03$ \\
\hline $\mathrm{B} 10$ & $1.5668 \mathrm{E}-02$ & & & & $8.1900 \mathrm{E}-03$ \\
\hline $\mathrm{B} 11$ & $6.3065 \mathrm{E}-02$ & & & & $3.2966 \mathrm{E}-02$ \\
\hline $\mathrm{Na}$ & & & $2.2272 \mathrm{E}-02$ & & $3.8576 \mathrm{E}-03$ \\
\hline $\mathrm{Fe}$ & & $6.9844 \mathrm{E}-02$ & & $6.9844 \mathrm{E}-02$ & $2.1237 \mathrm{E}-02$ \\
\hline $\mathrm{Ni}$ & & $4.3063 \mathrm{E}-04$ & & $4.3063 \mathrm{E}-04$ & $1.3094 \mathrm{E}-04$ \\
\hline $\mathrm{Cr}$ & & $1.0385 \mathrm{E}-02$ & & $1.0385 \mathrm{E}-02$ & $3.1577 \mathrm{E}-03$ \\
\hline $\mathrm{Mn55}$ & & $4.6007 \mathrm{E}-04$ & & $4.6007 \mathrm{E}-04$ & $1.3989 \mathrm{E}-04$ \\
\hline $\mathrm{Mo}$ & & $4.9097 \mathrm{E}-04$ & & $4.9097 \mathrm{E}-04$ & $1.4929 \mathrm{E}-04$ \\
\hline
\end{tabular}

12. Outside core

\begin{tabular}{|l|l|l|l|}
\hline \multicolumn{3}{|l|}{ Heterogeneous } & \multirow{2}{*}{ Homogeneous } \\
\hline & Barrel & Coolant & \\
\hline $\mathrm{Na}$ & & $2.22724 \mathrm{E}-02$ & $1.81469 \mathrm{E}-02$ \\
\hline $\mathrm{Fe}$ & $5.20478 \mathrm{E}-02$ & & $9.64075 \mathrm{E}-03$ \\
\hline $\mathrm{Ni}$ & $1.06872 \mathrm{E}-02$ & & $1.97959 \mathrm{E}-03$ \\
\hline $\mathrm{Cr}$ & $1.06058 \mathrm{E}-02$ & & $1.96450 \mathrm{E}-03$ \\
\hline $\mathrm{Mn} 55$ & $1.66670 \mathrm{E}-03$ & & $3.08720 \mathrm{E}-04$ \\
\hline $\mathrm{Mo}$ & $1.42668 \mathrm{E}-03$ & & $2.64263 \mathrm{E}-04$ \\
\hline
\end{tabular}




\section{Argonne}

\section{Nuclear S Engineering Division}

Argonne National Laboratory

9700 South Cass Avenue

Argonne, IL 60439

www.anl.gov 